\title{
FBXL19-AS1 promotes the progression of nasopharyngeal carcinoma by acting as a competing endogenous RNA to sponge miR-431 and upregulate PBOV1
}

\author{
HONGJUN DONG ${ }^{*}$, CHAO HUANG ${ }^{*}$ and JINGJING HUANG \\ Department of Otorhinolaryngology, Zhangjiagang TCM Hospital Affiliated to \\ Nanjing University of Chinese Medicine, Zhangjiagang, Jiangsu 215600, P.R. China
}

Received December 24, 2020; Accepted April 1, 2021

DOI: $10.3892 / \mathrm{mmr} .2021 .12286$

\begin{abstract}
Long non-coding RNAs (lncRNAs) have been shown to function as crucial regulators in the progression of various types of cancer, including nasopharyngeal carcinoma (NPC). The aim of the present study was to investigate the mechanisms underlying the role of the FBXL19-AS1/microRNA (miR)-431/prostate and breast cancer overexpressed 1 (PBOV1) axis in the progression of NPC. The expression levels of FBXL19-AS1, miR-431 and PBOV1 were assessed by reverse transcription-quantitative PCR. The Cell Counting Kit-8 assay was utilized to detect cell viability. Cell migration and invasion were determined using a Transwell assay. The associations between FBXL19-AS1 and miR-431 or miR-431 and PBOV1 were verified via bioinformatics analysis, dual-luciferase and RNA-binding protein immunoprecipitation assays. It was demonstrated that the expression levels of FBXL19-AS1 and PBOV1 were upregulated in NPC tissues and cells, whereas miR-431 expression was downregulated. FBXL19-AS1 directly interacted with miR-431. FBXL19-AS1 silencing inhibited the viability, migration and invasion of C666-1 and SUNE1 cells, whereas these effects could be alleviated by suppressing miR-431. miR-431 could target the 3'-untranslated region of PBOV1. Overexpression of PBOV1 neutralized the miR-431-mediated suppression of NPC progression. Moreover, FBXL19-AS1 could regulate PBOV1 by sponging miR-431 in NPC cells. In conclusion, the 1ncRNA FBXL19-AS1 accelerated NPC progression via the miR-431/PBOV1 axis, suggesting that it
\end{abstract}

Correspondence to: Dr Chao Huang, Department of Otorhinolaryngology, Zhangjiagang TCM Hospital Affiliated to Nanjing University of Chinese Medicine, 77 Changannan Road, Zhangjiagang, Jiangsu 215600, P.R. China

E-mail: huangchao_526@163.com

${ }^{*}$ Contributed equally

Key words: FBXL19-AS1, microRNA-431, prostate and breast cancer overexpressed 1, nasopharyngeal carcinoma may serve as a potential therapeutic target for patients with NPC.

\section{Introduction}

Nasopharyngeal carcinoma (NPC) is a malignant and aggressive tumor originating in the nasopharyngeal epithelium, which is characterized by a high incidence rate in Southern China (1-3). Despite the great advances achieved in the treatment of NPC, patients with NPC generally have a poor prognosis (4). Previous studies indicated that Epstein-Barr virus infection is a major pathogenic factor for NPC (5). With the development of second-generation sequencing, a large number of genes or non-protein-coding transcripts, such as long non-coding RNAs (lncRNAs) and microRNAs (miRNAs/miRs) have been identified in NPC, and they may serve as biomarkers for the diagnosis, treatment and prognosis of NPC (6-8).

lncRNAs are a family of non-coding RNAs that are $>200$ nucleotides (nt) in length and lack protein-coding ability (9). Accumulating evidence has demonstrated the regulatory functions of lncRNAs in multiple types of cancer, including NPC (10). For example, the depletion of ZFAS1 suppressed the development of NPC by regulating miR-135a (11). The knockdown of SOX2 overlapping transcript suppressed the viability, migration and invasion of NPC via the miR-146b-5p/heterogeneous nuclear ribonucleoproteins A2/B1 axis (12). Small nucleolar RNA host gene 5 accelerated NPC progression by adsorbing miR-1179 (13). DLX6-AS1 upregulated the level of hypoxia-inducible factor- $1 \alpha$ and facilitated the progression of NPC via sponging miR-199a-5p (14). FBXL19-AS1 has been identified as an oncogene in various human cancers, such as colorectal (15), lung (16) and breast (17) cancers. However, the function of FBXL19-AS1 in NPC remains unknown.

miRNAs are small non-coding RNAs that are $22 \mathrm{nt}$ in length $(18,19)$. Numerous studies have revealed that the aberrant expression of miRNAs is implicated in the progression of various tumors, including NPC $(20,21)$. For example, miR-449b-5p inhibited cell viability, migration and invasion by regulating tumor protein D52 in NPC (22). miR-432 repressed the migration and invasion of NPC cells by modulating transcription factor E2F3 expression (23). miR-100 suppressed cell viability and growth by modulating homeobox protein 
Hox-A1 expression (24). miR-431 has been reported to inhibit the development and progression of various cancers, such as melanoma (25), hepatocellular carcinoma (26) and papillary thyroid carcinoma (27). However, the involvement of miR-431 in the development of NPC remains unclear.

The present study was undertaken to investigate whether FBXL19-AS1 contributes to the progression of NPC via the miR-431/prostate and breast cancer overexpressed 1 (PBOV1) axis, in the hope that the findings may uncover novel targets for NPC treatment.

\section{Materials and methods}

Clinical specimens. A total of 30 NPC and 30 adjacent normal tissue samples (distance from tumor margin, $2 \mathrm{~cm}$ ) were obtained from patients with NPC (age range, 33-67 years) at Zhangjiagang TCM Hospital Affiliated to Nanjing University of Chinese Medicine (Zhangjiagang, China) between March 2016 and September 2019. The inclusion criteria were as follows: Patients were diagnosed as NPC with no history of other tumor. The exclusion criteria were as follows: i) diagnosed with other diseases; and ii) patients who had received preoperative radiotherapy, chemotherapy or other adjuvant treatments prior to admission. All fresh specimens were immediately frozen in liquid nitrogen and stored at $-80^{\circ} \mathrm{C}$ until RNA extraction. Written informed consent was obtained from each participant. The protocol of the present study was approved by the ethics committee of Zhangjiagang Hospital of Traditional Chinese Medicine. The clinical information of patients with NPC is presented in Table I.

Cell culture. Human NPC cell lines (C666-1, SUNE1, 5-8F and 6-10B) and nasopharyngeal epithelial cells (NP69) were obtained from Shanghai Institutes for Biological Sciences, Chinese Academy of Sciences. All cells were cultured in RPMI-1640 medium (Thermo Fisher Scientific, Inc.) supplemented with 10\% FBS (Invitrogen; Thermo Fisher Scientific, Inc.) at $37^{\circ} \mathrm{C}$ with $5 \% \mathrm{CO}_{2}$.

Cell transfection. Short hairpin RNA (shRNA) targeting FBXL19-AS1 (sh-FBXL19-AS1; 5'-GCAGGCCCAUCUACG GUGUGU-3') and control (sh-NC; 5'-AAUUAGCCGCAU GCGUCACAU-3'), miR-431 mimics (5'-UGCAAUGUUAGA UGGUGUGAGG-3'), negative control (NC mimics; 5'-GAC UCCUUACUCGCUCUACUG-3'), miR-431 inhibitor (5'-UUC ACCGGAUCUGUCACGUAU-3') and control (NC inhibitor; 5'-CAGUAGAGAUUGAAAGUUGUC-3') were obtained from Shanghai GenePharma Co., Ltd. To establish FBXL19-AS1 or PBOV1-overexpression plasmids (pcDNA3.1-FBXL19-AS1 or pcDNA3.1-PBOV1), the full-length FBXL19-AS1 or PBOV1 sequence was inserted into pcDNA3.1 vector (Thermo Fisher Scientific, Inc.), with pcDNA3.1 as a control. Lipofectamine ${ }^{\circledR}$ 2000 reagent (Invitrogen; Thermo Fisher Scientific, Inc.) was used for transfection of C666-1 and SUNE1 cells (1x10 $)$ with sh-FBXL19-AS1 (10 nM), sh-NC (10 nM), miR-431 mimics (10 nM), NC mimics (10 nM), miR-431 inhibitor (10 nM), NC inhibitor (10 nM) pcDNA3.1-FBXL19-AS1 (10 nM), pcDNA3.1-PBOV1 (10 nM) or pcDNA3.1 (10 nM) at room temperature for $\sim 30 \mathrm{~min}$. At $48 \mathrm{~h}$ post-transfection, subsequent experiments were performed.
Reverse transcription-quantitative PCR (RT-qPCR) analysis. Total RNA was extracted from NPC tissues and cells using TRIzol ${ }^{\circledR}$ reagent (Invitrogen; Thermo Fisher Scientific, Inc.). Then, cDNA was established from total RNA using the PrimeScript $^{\mathrm{TM}}$ 1st strand cDNA Synthesis Kit (cat. no. 6110A; Takara Biotechnology Co., Ltd.) according to the manufacturer's protocol. qPCR was performed using SYBR Green Taq ReadyMix (Sigma-Aldrich; Merck KGaA) on an Applied Biosystems 7500 Real-Time PCR System (Applied Biosystems; Thermo Fisher Scientific, Inc.). The following thermocycling conditions were used for qPCR: Initial denaturation at $95^{\circ} \mathrm{C}$ for $15 \mathrm{sec} ; 40$ cycles of denaturation at $94^{\circ} \mathrm{C}$ for $30 \mathrm{sec}$, annealing at $60^{\circ} \mathrm{C}$ for $20 \mathrm{sec}$ and extension at $72^{\circ} \mathrm{C}$ for $40 \mathrm{sec}$. The expression of genes was detected using the $2^{-\Delta \Delta \mathrm{Cq}}$ method (28). GAPDH or U6 were used as the internal controls for FBXL19-AS1 and PBOV1 or miR-431, respectively. The primer sequences were as follows: FBXL19-AS1 forward, 5'-GGTACAACTACGGATATGA-3' and reverse, 5'-TACGTC TCGACCATTACGCA-3'; miR-431 forward, 5'-TGTCTT GCAGGCCGTCATG-3' and reverse, 5'-GCTGTCAACGAT ACGCTACCTA-3'; PBOV1 forward, 5'-TGAGTCCCCTCT CGGTAATG-3' and reverse, 5'-GCCCCGAGTTAAGAA CATCA-3'; GAPDH forward, 5'-ACCACAGTCCATGCCATC AC-3' and reverse, 5'-TCCACCACCCTGTTGCTGTA-3'; and U6 forward, 5'-ATTGGAACGATACAGAGAAGATT-3' and reverse, 5'-GGAACGCTTCACGAATTTG-3'.

Cell Counting Kit-8 (CCK-8) assay. Cell viability was examined using a CCK-8 assay (Dojindo Molecular Technologies, Inc.). The transfected C666-1 and SUNE1 cells $\left(1 \times 10^{5}\right.$ cells/well) were plated into $96-$ well plates for $48 \mathrm{~h}$. Then, CCK-8 reagent was added to each well at $0,24,48$ and $72 \mathrm{~h}$ and incubated at $37^{\circ} \mathrm{C}$ for $4 \mathrm{~h}$. The absorbance at $450 \mathrm{~nm}$ was detected by a microplate reader (Olympus Corporation).

Transwell assay. The Transwell assay was performed using Transwell chambers (8.0- $\mu \mathrm{m}$ pore size; EMD Millipore). For the migration assay, transfected C666-1 and SUNE1 cells $\left(1 \times 10^{5}\right)$ in serum-free culture RPMI-1640 medium (Thermo Fisher Scientific, Inc.) were added to the upper chamber. Subsequently, RPMI-1640 medium with $10 \%$ FBS was added to the lower chamber. After $24 \mathrm{~h}, 4 \%$ paraformaldehyde and $0.1 \%$ crystal violet solution was used to fix and stain the cells, respectively, that had migrated to the lower surface of the membrane for $20 \mathrm{~min}$ at room temperature, and the cells were counted under a light microscope (magnification, x200). For cell invasion, the upper chamber was pre-coated with Matrigel for $1 \mathrm{~h}$ at room temperature. The remaining steps were consistent with those for the cell migration assay.

Dual-luciferase reporter assay. Starbase (version 2.0; starbase.sysu.edu.cn/) was used to predict the binding sites between FBXL19-AS1 and miR-431. TargetScan (www. targetscan.org/vert_72/) was used to predict the binding sites between miR-431 and PBOV1. The mutated 3'-UTR was generated using the QuikChange II Site Directed Mutagenesis kit (Agilent Technologies, Inc.). The 3'-untranslated region (UTR) of wild-type (WT) and mutant (Mut) FBXL19-AS1 (WT-FBXL19-AS1 and Mut-FBXL19-AS1) or PBOV1 (WT-PBOV1 and Mut-PBOV1) were inserted into the 
Table I. Clinicopathological characteristics of patients with nasopharyngeal carcinoma.

\begin{tabular}{lc}
$\begin{array}{l}\text { Clinicopathological } \\
\text { characteristics }\end{array}$ & $\begin{array}{c}\text { Number } \\
\text { of patients }(\mathrm{n}=30)\end{array}$ \\
\hline Age, years & 14 \\
$<50$ & 16 \\
$\geq 50$ & \\
Sex & 15 \\
Male & 15 \\
Female & \\
Tumor size, cm & 12 \\
$<5$ & 18 \\
$\geq 5$ & \\
Lymph node status & 11 \\
N0 & 19 \\
N1-3 & \\
Distant metastasis & 10 \\
No & 20 \\
Yes & \\
TNM stage & 6 \\
I-II & 24 \\
III-IV & \\
\hline
\end{tabular}

TNM, tumor node metastasis.

pmiR-GLO vector (Promega Corporation). Then, all plasmids $(0.6 \mu \mathrm{g})$ were transfected into C666-1 and SUNE1 cells $\left(1 \times 10^{5}\right)$ with $10 \mathrm{nM}$ miR-431 mimics or $10 \mathrm{nM}$ NC mimics using Lipofectamine 2000 (Invitrogen; Thermo Fisher Scientific, Inc.). After $48 \mathrm{~h}$, the luciferase activities were detected using the Dual-Luciferase Reporter System (Promega Corporation). Firefly luciferase activity was normalized to Renilla luciferase gene activity.

RNA-binding protein immunoprecipitation (RIP) assay. A RIP assay was utilized with Magna RIP RNA-Binding Protein Immunoprecipitation Kit (EMD Millipore). The transfected C666-1 and SUNE1 cells were dissolved in RIP lysis buffer (Beyotime Institute of Biotechnology), and then cell lysate was centrifuged at $40,000 \mathrm{x} \mathrm{g}$ at $4^{\circ} \mathrm{C}$ for $10 \mathrm{~min}$ and incubated with $50 \mu \mathrm{l}$ magnetic beads bound with the $5 \mu \mathrm{g}$ Ago2 antibody (cat. no. 2897; Cell Signaling Technology, Inc.). IgG (5 $\mu \mathrm{g}$; cat. no. PP64B; EMD Millipore) was used as a control group. Then, immunoprecipitated RNA was detected via an RT-qPCR assay.

Statistical analysis. All experiments were repeated three times. All data were analyzed using GraphPad Prism 7 (GraphPad Software, Inc.) and presented as the mean \pm SD. Paired/unpaired Student's t-test or one-way ANOVA, followed by Tukey's post hoc test were used to evaluate the differences. The correlation between FBXL19-AS1 and PBOV1 expression was assessed by a Pearson's correlation analysis. $\mathrm{P}<0.05$ was considered to indicate a statistically significant difference.

\section{Results}

FBXL19-AS1 is significantly upregulated, whereas miR-431 is downregulated in NPC tissues and cells. To explore the function of FBXL19-AS1 and miR-431 (derived from 5' of pre-miR-431) in NPC, RT-qPCR was performed to detect the levels of FBXL19-AS1 and miR-431 in NPC tissues. The data indicated that FBXL19-AS1 was increased and miR-431 was decreased in NPC tissues ( $n=30$; Fig. 1A and B), and that FBXL19-AS1 expression was inversely correlated with miR-431 expression (Fig. 1C). Moreover, the levels of FBXL19-AS1 and miR-431 in NPC cell lines (C666-1, SUNE1, 5-8F and 6-10B) and NP69 cells were detected. Consistent with previous results, FBXL19-AS1 expression was upregulated in NPC cells, whereas miR-431 expression was downregulated, particularly in C666-1 and SUNE1 cell lines (Fig. 1D and E). These data suggested that FBXL19-AS1 may act as an oncogene and miR-431 as a tumor suppressor in the progression of NPC. Since C666-1 and SUNE1 exhibited the highest and lowest expression of FBXL19-AS1 and miR-431, respectively, these two cell lines were selected for subsequent experiments.

FBXL19-AS1 interacts with miR-431. A growing body of evidence has demonstrated that IncRNAs can interact with miRNAs to modulate cancer development $(29,30)$. In the present study, it was observed that FBXL19-AS1 acted as a molecular sponge for miR-431 (Fig. 2A). Moreover, the luciferase activity of FBXL19-AS1-WT was reduced by miR-431 mimics in C666-1 and SUNE1 cells, whereas the activity of FBXL19-AS1-Mut was not changed (Fig. 2B). Furthermore, the RIP assay revealed that FBXL19-AS1 and miR-431 were markedly enriched in the Ago2 group compared with the IgG group (Fig. 2C). To confirm whether FBXL19-AS1 could modulate the level of miR-431, pcDNA3.1-FBXL19-AS1 or sh-FBXL19-AS1 was transfected into C666-1 and SUNE1 cells to overexpress or suppress FBXL19-AS1, respectively (Fig. 2D), following which the expression of miR-431 was determined. The results indicated that overexpression of FBXL19-AS1 suppressed miR-431 expression and depletion of FBXL19-AS1 enhanced miR-431 expression (Fig. 2E). These results suggested that FBXL19-AS1 interacts with and negatively regulates the level of miR-431.

FBXL19-AS1 depletion suppresses the progression of $N P C$. To further explore the function of FBXL19-AS1 in the development of NPC, C666-1 and SUNE1 cells were transfected with sh-FBXL19-AS1 or sh-NC. The CCK-8 assay indicated that the interference of FBXL19-AS1 led to the inhibition of C666-1 and SUNE1 cell viability (Fig. 3A). Moreover, FBXL19-AS1 depletion markedly suppressed the migration and invasion of NPC cells (Fig. 3B and C). Thus, FBXL19-AS1 knockdown may inhibit the occurrence of NPC.

FBXL19-AS1 overexpression counteracts the effects of miR-431 on NPC cells. To investigate whether pcDNA3.1-FBXL19-AS1 can abolish the inhibition of NPC progression mediated by miR-431 mimics, the expression of miR-431 was assessed in C666-1 and SUNE1 cells transfected 
A

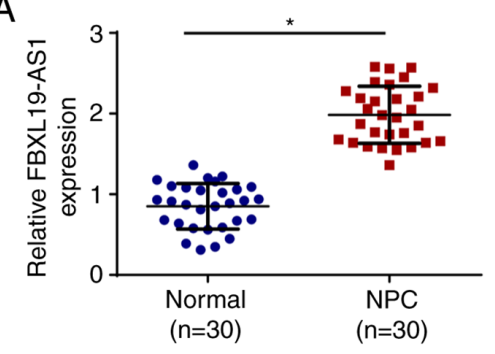

$\mathrm{B}$.

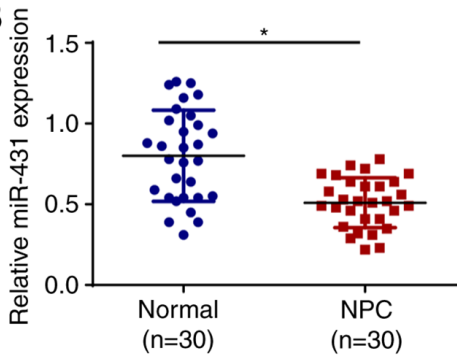

C

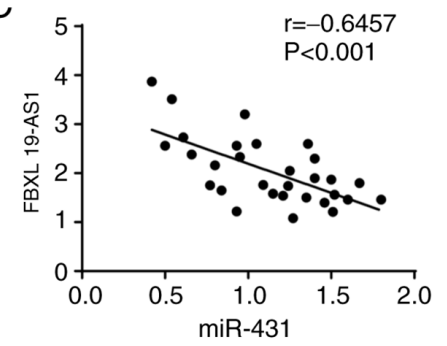

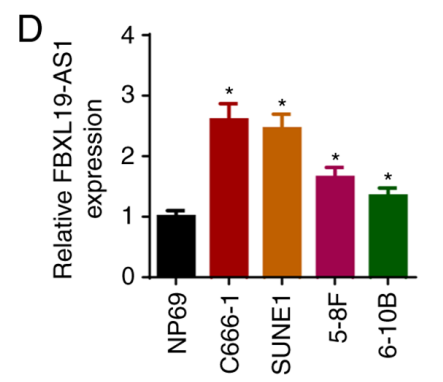

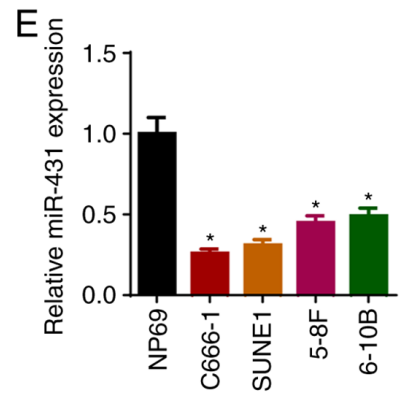

Figure 1. FBXL19-AS1 is significantly upregulated, whereas miR-431 is downregulated in NPC tissues and cells. The expression levels of (A) FBXL19-AS1 and (B) miR-431 in NPC tissues $(n=30)$ and adjacent normal tissues $(n=30)$ were measured via RT-qPCR. ${ }^{*} P<0.05$ as indicated. (C) The correlation between FBXL19-AS1 and miR-431 expression was detected by Pearson's correlation analysis. The expression levels of (D) FBXL19-AS1 and (E) miR-431 in NPC cells were detected via RT-qPCR. "P<0.05 vs. NP69 cells. miR, microRNA; NPC, nasopharyngeal carcinoma; RT-qPCR, reverse transcription-quantitative PCR.

A Mut FBXL19-AS1: 5' UAGAUGACAG -- CAUCGUCU $3^{\prime}$ WT FBXL19-AS1: 5' UAGAUGACAG -- AGCAAGAC 3' hsa-miR-431: 3' ACGUACUGCCGGACGUUCUG 5'

B

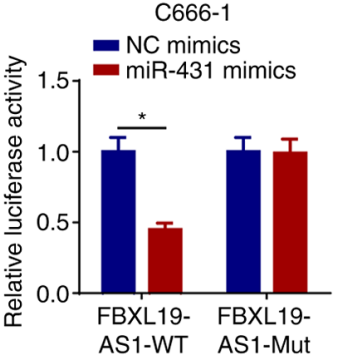

SUNE1

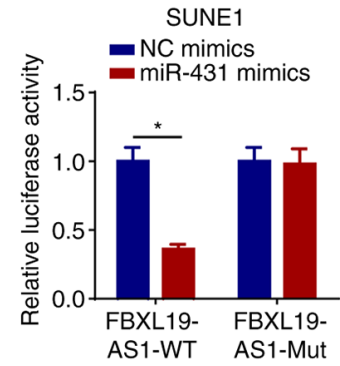

C

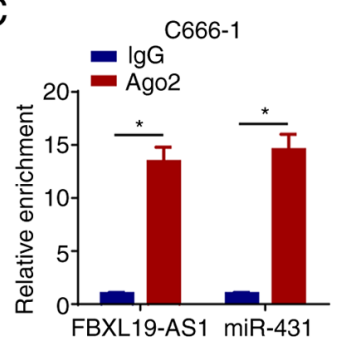

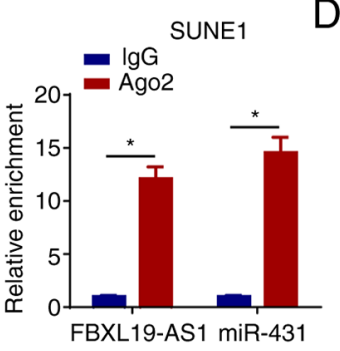

D

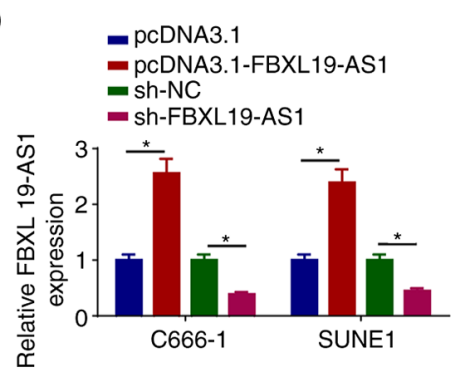

E

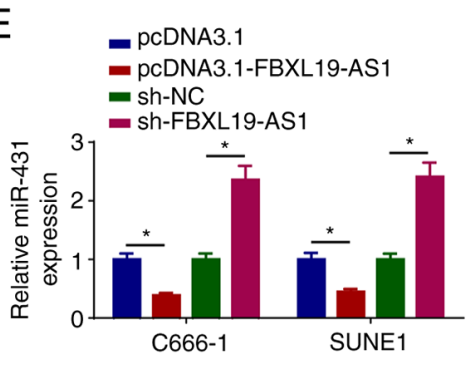

Figure 2. FBXL19-AS1 interacts with miR-431. (A) The predicted binding sites of FBXL19-AS1 and miR-431. (B) The luciferase activity of C666-1 and SUNE1 cells co-transfected with the miR-431 mimics and FBXL19-AS1-WT or FBXL19-AS1-Mut was detected. (C) The interaction between FBXL19-AS1 and miR-431 were detected via a RNA-binding protein immunoprecipitation assay. The expression levels of (D) FBXL19-AS1 and (E) miR-431 in C666-1 and SUNE1 cells transfected with pcDNA3.1-FBXL19-AS1 or sh-FBXL19-AS1 were detected via reverse transcription-quantitative PCR. "P<0.05. miR, microRNA; WT, wild-type; Mut, mutant; sh, short hairpin RNA; NC, negative control.

with NC mimics, miR-431 mimics, miR-431 mimics + pcDNA3.1, and miR-431 mimics + pcDNA3.1-FBXL19-AS1. The results indicated that the addition of FBXL19-AS1 diminished miR-431 expression (Fig. 4A). Further studies revealed that the overexpression of miR-431 inhibited the viability, migration and invasion of C666-1 and SUNE1 cells, which was partially reversed by pcDNA3.1-FBXL19-AS1 transfection (Fig. 4B-D). These results suggested that the
FBXL19-AS1/miR-431 axis may be implicated in NPC progression.

PBOVI is directly targeted by miR-431. A putative miR-431 binding site located in the 3'-UTR of PBOV1 mRNA was predicted by TargetScan (Fig. 5A). As shown in Fig. 5B, miR-431 mimics led to a significant reduction in relative luciferase activity of PBOV1-WT, but not in PBOV1-Mut. miR-431 and 
A
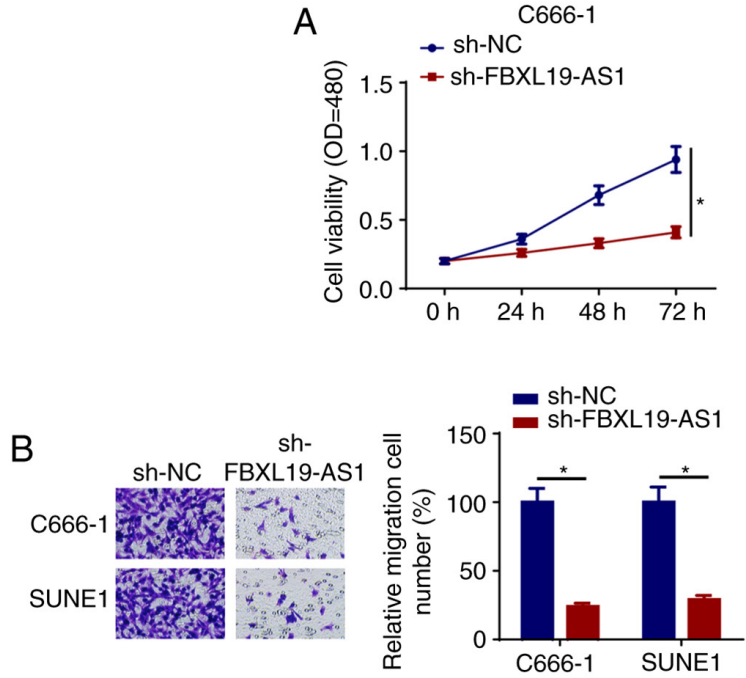
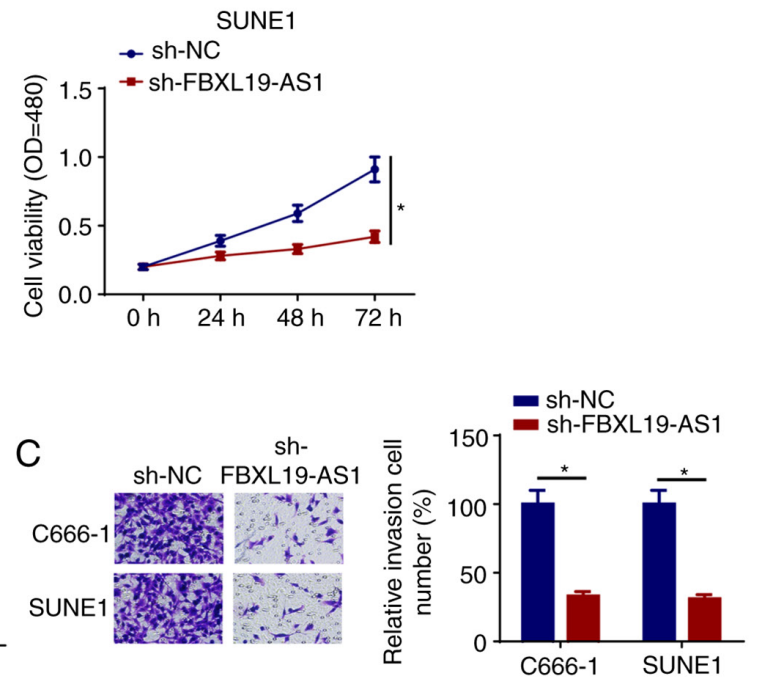

Figure 3. FBXL19-AS1 knockdown suppresses the progression of nasopharyngeal carcinoma. (A) Cell Counting Kit-8 assay was performed to detect cell viability of C666-1 and SUNE1 cells transfected with sh-FBXL19-AS1 or sh-NC. Transwell assay was used to detect cell (B) migration and (C) invasion of C666-1 and SUNE1 cells transfected with sh-FBXL19-AS1 or sh-NC. "P<0.05. miR, microRNA; sh, short hairpin RNA; NC, negative control.
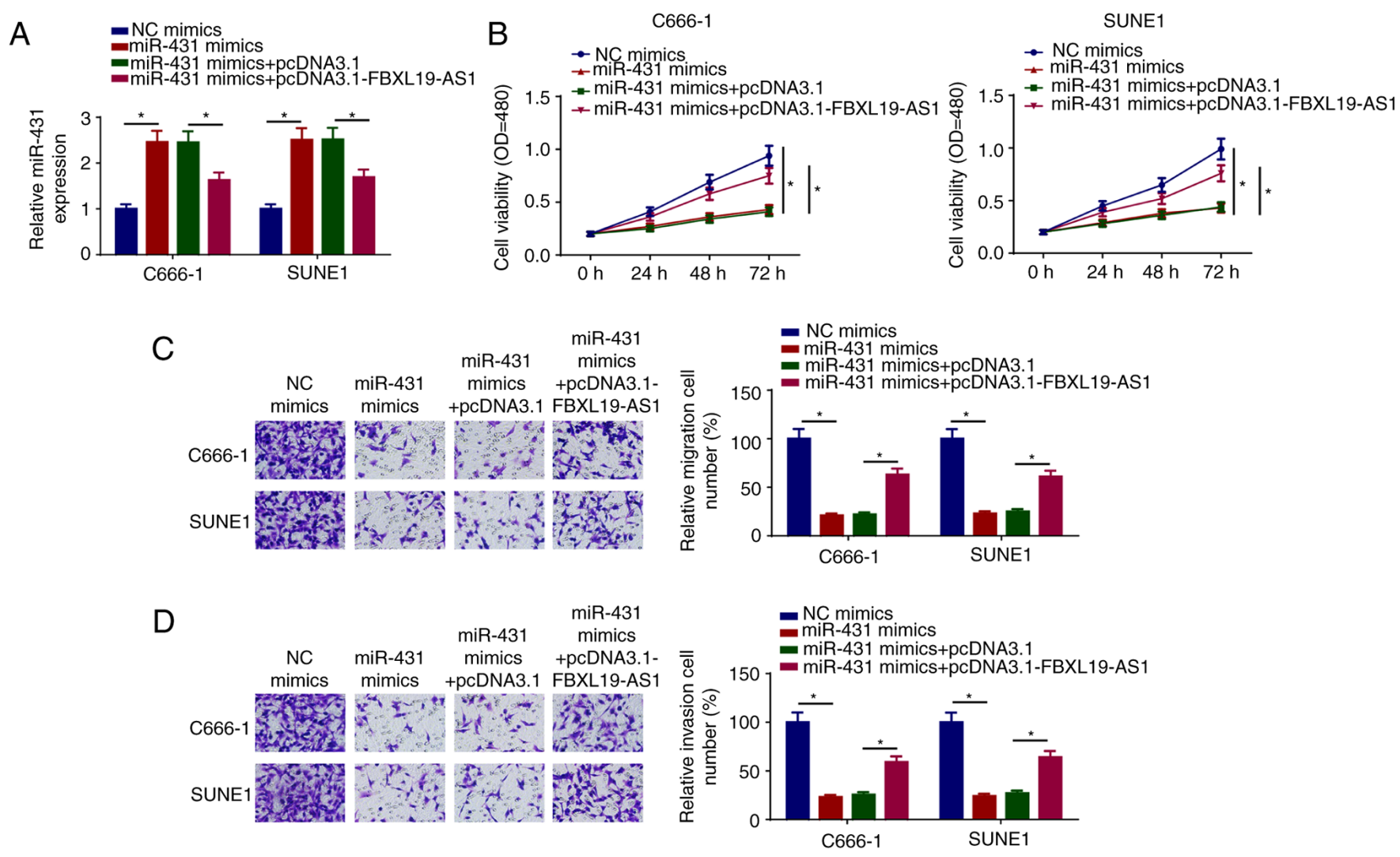

Figure 4. FBXL19-AS1 overexpression partially reverses the effects of miR-431 on nasopharyngeal carcinoma cells. (A) The expression levels of miR-431 in C666-1 and SUNE1 cells transfected with NC mimics, miR-431 mimics, miR-431 mimics + pcDNA3.1 and miR-431 mimics + pcDNA3.1-FBXL19-AS1 were detected via reverse transcription-quantitative PCR. (B) Cell Counting Kit-8 assay was used to detect the viability of C666-1 and SUNE1 cells transfected with NC mimics, miR-431 mimics, miR-431 mimics + pcDNA3.1 and miR-431 mimics + pcDNA3.1-FBXL19-AS1. Transwell assay was performed to measure (C) migration and (D) invasion of C666-1 and SUNE1 cells transfected with NC mimics, miR-431 mimics, miR-431 mimics + pcDNA3.1 and miR-431 mimics + pcDNA3.1-FBXL19-AS1. ${ }^{*} \mathrm{P}<0.05$. miR, microRNA; NC, negative control.

PBOV1 were markedly enriched by Ago2, while IgG enrichment was not obvious (Fig. 5C). Next, RT-qPCR results showed that miR-431 expression was reduced by transfecting miR-431 inhibitor in NPC cells (Fig. 5D). Moreover, the overexpression of miR-431 inhibited PBOV1 expression and the knockdown of
miR-431 enhanced PBOV1 expression (Fig. 5E). Furthermore, PBOV1 was markedly enhanced in NPC tissues compared with normal tissues (Fig. 5F). In addition, PBOV1 expression was negatively correlated with miR-431 expression (Fig. 5G). These results suggested that PBOV1 is a direct target of miR-431. 
A
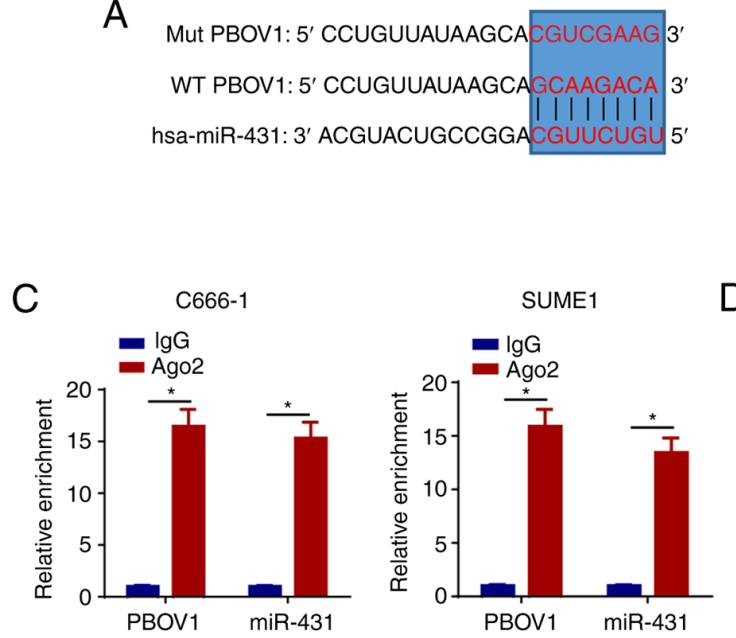

$\mathrm{F}$

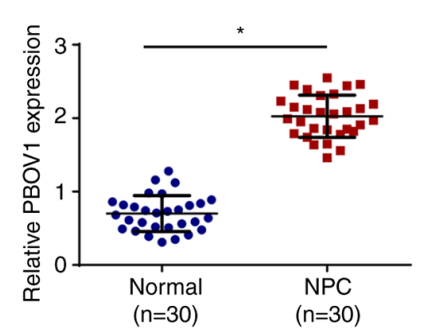

C666-1

B

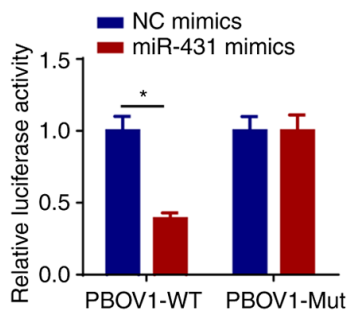

SUNE1

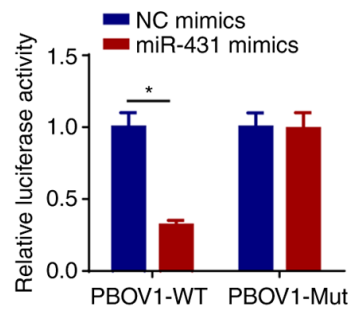

D

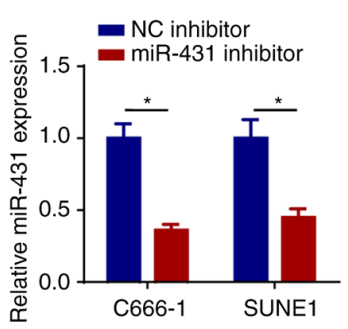

$E$

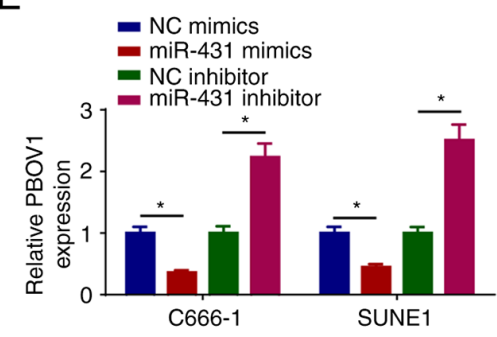

G

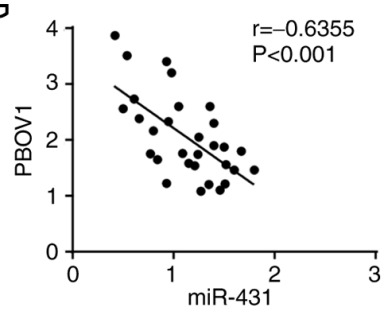

Figure 5. PBOV1 is directly targeted by miR-431. (A) The putative binding sites of miR-431 and PBOV1. (B) The luciferase activity of C666-1 and SUNE1 cells co-transfected with the miR-431 mimics and PBOV1-WT or PBOV1-Mut was detected. (C) The RNA-binding protein immunoprecipitation assay was utilized to analyze the interaction between miR-431 and PBOV1. (D) The expression of miR-431 in NPC cells transfected with NC inhibitor and miR-431 inhibitor was detected via RT-qPCR. (E) The expression of PBOV1 in C666-1 and SUNE1 cells transfected with miR-431 mimics or miR-431 inhibitor were detected via RT-qPCR. (F) The expression of PBOV1 in NPC tissues $(n=30)$ and adjacent normal tissues $(n=30)$ were measured via RT-qPCR. (G) The correlation between PBOV1 and miR-431 expression was analyzed using Pearson's correlation analysis. ${ }^{*} \mathrm{P}<0.05$. PBOV1, prostate and breast cancer overexpressed 1 ; miR, microRNA; WT, wild-type; Mut, mutant; NC, negative control; RT-qPCR, reverse transcription-quantitative PCR; NPC, nasopharyngeal carcinoma.

FBXL19-AS1 regulates PBOV1 by sponging miR-431 in NPC cells. To further study the association between PBOV1 and miR-431 inNPC, the expression of PBOV1 was detected in C666-1 cells transfected with NC mimics, miR-431 mimics, miR-431 mimics + pcDNA3.1 and miR-431 mimics + pcDNA3.1-PBOV1. The transfection efficiency of PBOV1-overexpression plasmid was confirmed by observing the upregulation of PBOV1 in C666-1 cells (Fig. 6A). pcDNA3.1-PBOV1 partially reversed the suppressive effects of miR-431 mimics on PBOV1 expression (Fig. 6B). Moreover, the overexpression of miR-431 suppressed the viability, migration and invasion of C666-1 cells, which was counteracted following pcDNA3.1-PBOV1 transfection (Fig. 6C-E). FBXL19-AS1 expression was positively correlated with PBOV1 expression (Fig. 6F). Furthermore, C666-1 cells were treated with sh-NC, sh-FBXL19-AS1, sh-FBXL19-AS1 $+\mathrm{NC}$ inhibitor and sh-FBXL19-AS1 + miR-431 inhibitor, and the expression of PBOV1 was detected in transfected cells. The results demonstrated that FBXL19-AS1 knockdown decreased the expression of PBOV1, whereas these effects could be abolished by miR-431 inhibitor transfection in NPC cells (Fig. 6G). Taken together, the aforementioned results indicated that FBXL19-AS1 may drive the progression of NPC via modulation of the miR-431/PBOV1 axis.

\section{Discussion}

Previous studies have revealed that aberrant expression levels of lncRNAs are involved in the progression of various malignancies, including NPC $(31,32)$. The present study demonstrated that FBXL19-AS1 interference may suppress the progression of NPC via sponging miR-431 and inhibiting PBOV1. To the best of our knowledge, the present study was the first to demonstrate that the FBXL19-AS1/miR-431/PBOV1 axis may be implicated in the regulation of NPC.

FBXL19-AS1 has been identified as an oncogene in various cancers. For example, FBXL19-AS1 accelerated cell viability and suppressed cell apoptosis by regulating miR-876-5p in breast cancer (33). FBXL19-AS1 accelerated the progression of lung adenocarcinoma by inhibiting miR-203a (34). In the present study, FBXL19-AS1 was found to be upregulated in NPC tissues and cells. Functionally, the depletion of FBXL19-AS1 inhibited the viability, migration and invasion of NPC cells. These results indicated that FBXL19-AS1 may function as an oncogene in NPC.

lncRNAs may act as competing endogenous RNAs (ceRNAs) by specifically adsorbing miRNAs, and then regulating the levels of target genes $(35,36)$. Multiple lncRNAs, 
A

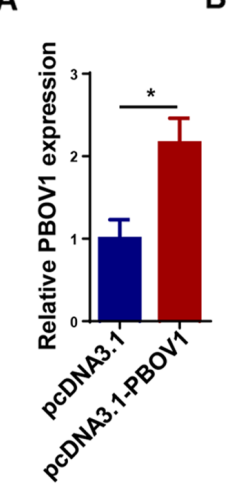

D

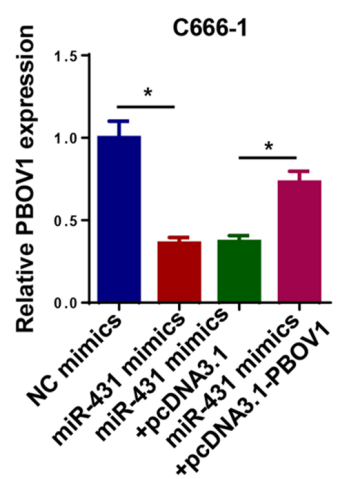

C
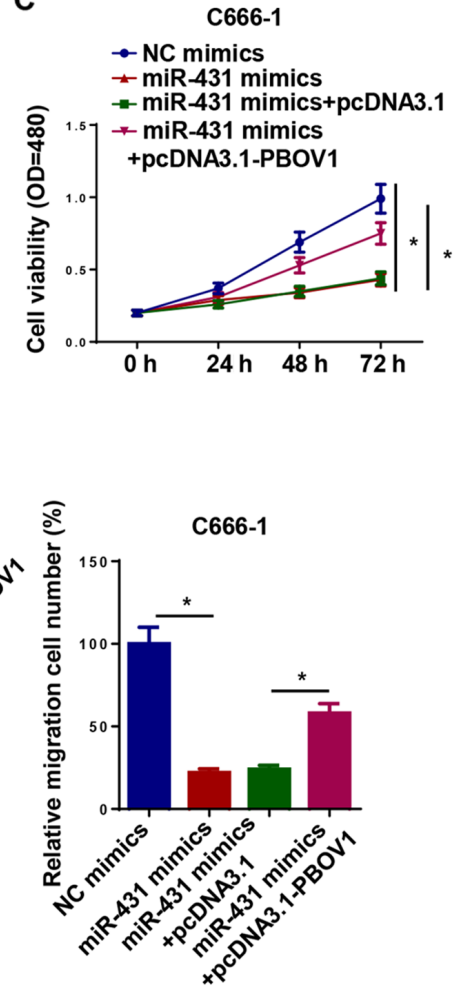

E
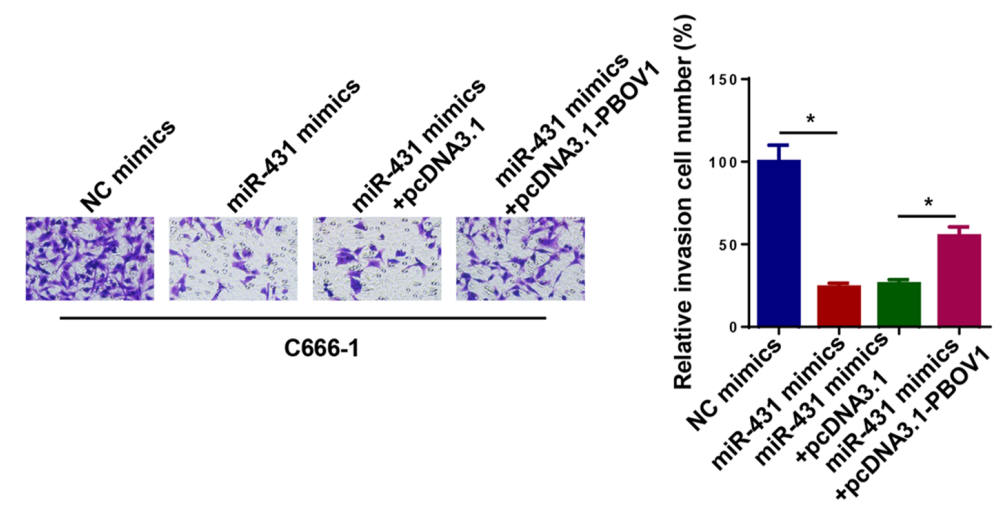

$\mathbf{F}$

G
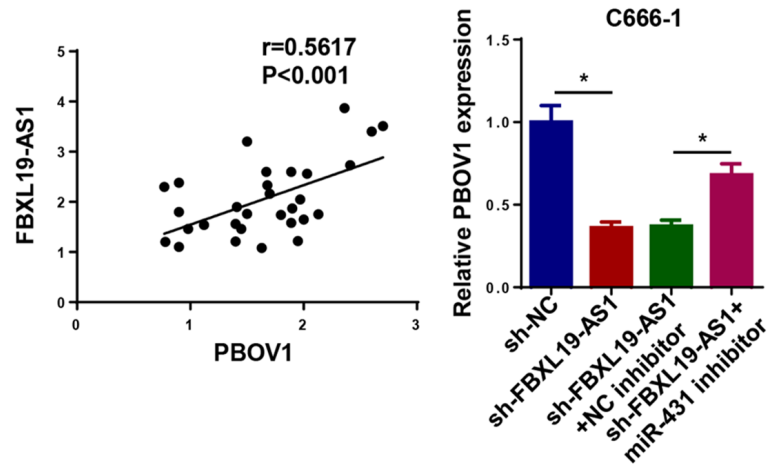

Figure 6. FBXL19-AS1 regulates PBOV1 by sponging miR-431 in nasopharyngeal carcinoma cells. (A) The expression of PBOV1 in C666-1 cells transfected with pcDNA3.1 and pcDNA3.1-PBOV1 was detected via RT-qPCR. (B) The expression of PBOV1 in C666-1 cells transfected with NC mimics, miR-431 mimics, miR-431 mimics + pcDNA3.1 and miR-431 mimics + pcDNA3.1-PBOV1 was detected via RT-qPCR. (C) Cell Counting Kit-8 assay was used to detect the viability of C666-1 cells transfected with NC mimics, miR-431 mimics, miR-431 mimics + pcDNA3.1 and miR-431 mimics + pcDNA3.1-PBOV1. Transwell assay was performed to measure the (D) migration and (E) invasion of C666-1 cells transfected with NC mimics, miR-431 mimics, miR-431 mimics + pcDNA3.1 and miR-431 mimics + pcDNA3.1-PBOV1. (F) The correlation between PBOV1 and FBXL19-AS1 expression was analyzed using Pearson's correlation analysis. (G) The expression of PBOV1 in C666-1 cells transfected with sh-NC, sh-FBXL19-AS1, sh-FBXL19-AS1 + NC inhibitor and sh-FBXL19-AS1 + miR-431 inhibitor was detected via RT-qPCR. "P<0.05. PBOV1, prostate and breast cancer overexpressed 1; miR, microRNA; NC, negative control; RT-qPCR, reverse transcription-quantitative PCR; sh, short hairpin RNA. 
such as PVT1 (37), ZNF667-AS1 (38) and MSC-AS1 (39), have been reported to serve as ceRNAs in the development of NPC. The present study confirmed that miR-431 contained predicted binding sites for FBXL19-AS1. It was previously demonstrated that miR-431 plays a key role in human malignancies. For example, miR-431 was found to suppress breast cancer progression via regulating fibroblast growth factor 9 (40). In addition, the upregulation of miR-431 markedly suppressed colon cancer development via targeting autophagy related 3 (41). Furthermore, miR-431 repressed lung cancer progression via regulating RAF proto-oncogene serine/threonine-protein kinase (16). In the present study, the expression of miR-431 was decreased in NPC tissues and cells, and it was a downstream target of FBXL19-AS1. The overexpression of FBXL19-AS1 inhibited miR-431 expression and interference of FBXL19-AS1 enhanced miR-431 expression. The transfection of miR-431 mimics suppressed the progression of NPC, which was counteracted following pcDNA3.1-FBXL19-AS1 transfection. The aforementioned data demonstrated that FBXL19-AS1 promoted the progression of NPC by regulating the expression of miR- 431 .

PBOV1 is a human protein-coding gene with a 2,501-bp single-exon mRNA (42). PBOV1 has been reported to be upregulated in several cancers. For example, the overexpression of PBOV1 accelerated the tumorigenicity of prostate cancer cells (43). PBOV1 facilitated the viability and metastasis of hepatocellular carcinoma cells in vitro (44). Moreover, PBOV1 may be valuable as a biomarker for the treatment of prostate cancer (45). In the present study, PBOV1 was directly targeted by miR-431 and PBOV1 expression was found to be negatively associated with miR-431 expression. Overexpression of miR-431 inhibited the viability, migration and invasion of NPC cells, whereas these effects could be abolished by upregulating PBOV1 in NPC. Furthermore, the suppressive effects of FBXL19-AS1 silencing on PBOV1 expression were partly neutralized by miR-431 inhibitor transfection in NPC cells.

However, there were several limitations to the present study. Firstly, there may be other downstream targets of miR-143, which may also act as crucial regulators in the pathogenesis of NPC. Secondly, increasing the patient sample size may further verify the conclusion that FBXL19-AS1 is highly expressed and acts as an oncogene in NPC.

To the best of our knowledge, the present study was the first to demonstrate the molecular mechanism underlying the role of FBXL19-AS1 in NPC. The findings demonstrated that FBXL19-AS1 was upregulated in NPC tissues and cells, and FBXL19-AS1 silencing suppressed viability, migration and invasion of NPC cells, which suggested the oncogenic role of FBXL19-AS1 in NPC. In addition, accumulating studies have indicated that lncRNAs can serve as tools in the diagnosis, treatment and prognosis of NPC $(46,47)$. Therefore, the FBXL19-AS1/miR-431/PBOV1 axis may be a novel promising therapeutic strategy for patients with NPC.

\section{Acknowledgements}

Not applicable.

\section{Funding}

No funding was received.

\section{Availability of data and materials}

The datasets used and/or analyzed during the present study are available from the corresponding author upon reasonable request.

\section{Authors' contributions}

$\mathrm{JH}$ performed the experiments. $\mathrm{HD}$ and $\mathrm{CH}$ analyzed the data and wrote the manuscript. All authors confirm the authenticity of all the raw data. All authors read and approved the final manuscript.

\section{Ethics approval and consent to participate}

Written informed consent was obtained from each participant. The protocol of the present study was approved by the Ethics Committee of Zhangjiagang TCM Hospital Affiliated to Nanjing University of Chinese Medicine (Zhangjiagang, China).

\section{Patient consent for publication}

Not applicable.

\section{Competing interests}

The authors declare that they have no competing interests.

\section{References}

1. Wei KR, Zheng RS, Zhang SW, Liang ZH, Li ZM and Chen WQ: Nasopharyngeal carcinoma incidence and mortality in China, 2013. Chin J Cancer 36: 90, 2017.

2. Zhang W, Guo Q, Liu G, Zheng F, Chen J, Huang D, Ding L, Yang X, Song E, Xiang Y and Yao H: NKILA represses nasopharyngeal carcinoma carcinogenesis and metastasis by NF-kB pathway inhibition. PLoS Genet 15: e1008325, 2019.

3. Razak AR, Siu LL, Liu FF, Ito E, O'Sullivan B and Chan K: Nasopharyngeal carcinoma: The next challenges. Eur J Cancer 46: 1967-1978, 2010.

4. Cao C, Zhou S, and Hu J: Long noncoding RNA MAGI2-AS3/miR-218-5p/GDPD5/SEC61A1 axis drives cellular proliferation and migration and confers cisplatin resistance in nasopharyngeal carcinoma. Int Forum Allergy Rhinol 10: 1012-1023, 2020.

5. Young LS and Dawson CW: Epstein-Barr virus and nasopharyngeal carcinoma. Chin J Cancer 33: 581-590, 2014.

6. Zhao CX, Zhu W, Ba ZQ, Xu HJ, Liu WD, Zhu B, Wang L, Song YJ, Yuan S and Ren CP: The regulatory network of nasopharyngeal carcinoma metastasis with a focus on EBV, lncRNAs and miRNAs. Am J Cancer Res 8: 2185-2209, 2018.

7. Wang Y, Chen W, Lian J, Zhang H, Yu B, Zhang M, Wei F, Wu J, Jiang J, Jia Y, et al: The lncRNA PVT1 regulates nasopharyngeal carcinoma cell proliferation via activating the KAT2A acetyltransferase and stabilizing HIF-1 $\alpha$. Cell Death Differ 27: 695-710, 2020.

8. Zheng ZQ, Li ZX, Zhou GQ, Lin L, Zhang LL, Lv JW, Huang XD, Liu RQ, Chen F, He XJ, et al: Long noncoding RNA FAM225A promotes nasopharyngeal carcinoma tumorigenesis and metastasis by acting as ceRNA to sponge miR-590-3p/miR-1275 and upregulate ITGB3. Cancer Res 79: 4612-4626, 2019.

9. Lou T, Ke K, Zhang L, Miao C and Liu Y: LncRNA PART1 facilitates the malignant progression of colorectal cancer via miR-150-5p/LRG1 axis. J Cell Biochem 121: 4271-4281, 2020.

10. Chen G, Wang Z, Wang D, Qiu C, Liu M, Chen X, Zhang Q, Yan G and Cui Q: LncRNADisease: A database for long-non-coding RNA-associated diseases. Nucleic Acids Res 41 (Database Issue): D983-D986, 2013. 
11. Wang M, Ji YQ, Song ZB, Ma XX, Zou YY and Li XS: Knockdown of lncRNA ZFAS1 inhibits progression of nasopharyngeal carcinoma by sponging miR-135a. Neoplasma 66 : 939-945, 2019.

12. Zhang E and Li X: LncRNA SOX2-OT regulates proliferation and metastasis of nasopharyngeal carcinoma cells through miR-146b-5p/HNRNPA2B1 pathway. J Cell Biochem 120: $16575-16588,2019$.

13. Liu D, Wang Y, Zhao Y and Gu X: LncRNA SNHG5 promotes nasopharyngeal carcinoma progression by regulating miR-1179/HMGB3 axis. BMC Cancer 20: 178, 2020.

14. Yang B, Jia L, Ren H, Jin C, Ren Q, Zhang H, Hu D, Zhang H, $\mathrm{Hu} \mathrm{L}$ and Xie T: LncRNA DLX6-AS1 increases the expression of HIF- $1 \alpha$ and promotes the malignant phenotypes of nasopharyngeal carcinoma cells via targeting MiR-199a-5p. Mol Genet Genomic Med 8: e1017, 2020.

15. Shen B, Yuan Y, Zhang Y, Yu S, Peng W, Huang X and Feng J: Long non-coding RNA FBXL19-AS1 plays oncogenic role in colorectal cancer by sponging miR-203. Biochem Biophys Res Commun 488: 67-73, 2017.

16. Jiang Q, Cheng L, Ma D and Zhao Y: FBXL19-AS1 exerts oncogenic function by sponging miR-431-5p to regulate RAF1 expression in lung cancer. Biosci Rep 39: BSR20181804, 2019.

17. Zhang Y, Xiao X, Zhou W, Hu J and Zhou D: LIN28A-stabilized FBXL19-AS1 promotes breast cancer migration, invasion and EMT by regulating WDR66. In Vitro Cell Dev Biol Anim 55: 426-435, 2019.

18. Fromm B, Billipp T, Peck LE, Johansen M, Tarver JE, King BL, Newcomb JM, Sempere LF, Flatmark K, Hovig E and Peterson KJ: A uniform system for the annotation of vertebrate microRNA Genes and the evolution of the human microRNAome. Annu Rev Genet 49: 213-242, 2015.

19. Rong X, Gao W, Yang $X$ and Guo J: Downregulation of hsa_circ_0007534 restricts the proliferation and invasion of cervical cancer through regulating miR-498/BMI-1 signaling. Life Sci 235: 116785, 2019.

20. Fridrichova I and Zmetakova I: MicroRNAs contribute to breast cancer invasiveness. Cells 8: 1361, 2019.

21. Aghajani M, Mansoori B, Mohammadi A, Asadzadeh Z and Baradaran B: New emerging roles of CD133 in cancer stem cell: Signaling pathway and miRNA regulation. J Cell Physiol 234 21642-21661, 2019.

22. Yin W, Shi L, and Mao Y: MicroRNA-449b-5p suppresses cell proliferation, migration and invasion by targeting TPD52 in nasopharyngeal carcinoma. J Biochem 166: 433-440, 2019.

23. Wang T, Du M, Zhang W, Bai H, Yin L, Chen W, He X and Chen Q: MicroRNA-432 suppresses invasion and migration via E2F3 in nasopharyngeal carcinoma. Onco Targets Ther 12 11271-11280, 2019.

24. He W, Huang Y, Jiang CC, Zhu Y, Wang L, Zhang W, Huang W, Zhou T and Tang S: miR-100 inhibits cell growth and proliferation by targeting HOXA1 in nasopharyngeal carcinoma. Onco Targets Ther 13: 593-602, 2020

25. Yin D, Wei G, Yang F and Sun X: Circular RNA has circ 0001591 promoted cell proliferation and metastasis of human melanoma via ROCK1/PI3K/AKT by targeting miR-431-5p. Hum Exp Toxicol 40: 310-324, 2021.

26. Li MF, Li YH, He YH, Wang Q, Zhang Y, Li XF, Meng XM, Huang C and Li J: Emerging roles of hsa_circ_0005075 targeting miR-431 in the progress of HCC. Biomed Pharmacother 99: 848-858, 2018

27. Liu Y,Li L, Liu Z, Yuan Q and Lu X: Downregulation of miR-431 expression associated with lymph node metastasis and promotes cell invasion in papillary thyroid carcinoma. Cancer Biomark 22: 727-732, 2018

28. Livak KJ and Schmittgen TD: Analysis of relative gene expression data using real-time quantitative PCR and the 2(-Delta Delta C(T)) method. Methods 25: 402-408, 2001.

29. Zhang Y, Qian W, Feng F, Cao Q, Li Y, Hou Y, Zhang L and Fan J: Upregulated lncRNA CASC2 may inhibit malignant melanoma development through regulating miR-18a-5p/RUNX1. Oncol Res 27: 371-377, 2019.
30. Gao W, Lin S, Cheng C, Zhu A, Hu Y, Shi Z, Zhang X and Hong Z: Long non-coding RNA CASC2 regulates Sprouty2 via functioning as a competing endogenous RNA for miR-183 to modulate the sensitivity of prostate cancer cells to docetaxel. Arch Biochem Biophys 665: 69-78, 2019.

31. Liu D, Gong H, Tao Z, Chen S, Kong Y and Xiao B: LncRNA IUR downregulates miR-144 to regulate PTEN in nasopharyngeal carcinoma. Arch Physiol Biochem: Aug 14, 2020 (Epub ahead of print)

32. Tang T, Yang L, Cao Y, Wang M, Zhang S, Gong Z, Xiong F, He Y, Zhou Y, Liao Q, et al: LncRNA AATBC regulates Pinin to promote metastasis in nasopharyngeal carcinoma. Mol Oncol 14: 2251-2270, 2020.

33. Dong G, Pan T, Zhou D, Li C, Liu J and Zhang J: FBXL19-AS1 promotes cell proliferation and inhibits cell apoptosis via miR-876-5p/FOXM1 axis in breast cancer. Acta Biochim Biophys Sin (Shanghai) 51: 1106-1113, 2019.

34. Wang L, Zhang X, Liu Y and Xu S: Long noncoding RNA FBXL19-AS1 induces tumor growth and metastasis by sponging miR-203a-3p in lung adenocarcinoma. J Cell Physiol 235: 3612-3625, 2020

35. Yang J, Qiu Q, Qian X, Yi J, Jiao Y, Yu M, Li X, Li J, Mi C, Zhang J, et al: Long noncoding RNA LCAT1 functions as a ceRNA to regulate RAC1 function by sponging miR-4715-5p in lung cancer. Mol Cancer 18: 171, 2019.

36. Thomson DW and Dinger ME: Endogenous microRNA sponges: Evidence and controversy. Nat Rev Genet 17: 272-283, 2016.

37. Cui M, Chang Y, Fang QG, Du W, Wu JF, Wang JH, Liu ST and Luo SX: Non-Coding RNA Pvt1 promotes cancer stem cell-like traits in nasopharyngeal cancer via inhibiting miR-1207. Pathol Oncol Res 25: 1411-1422, 2019.

38. Chen X, Huang Y, Shi D, Nie C, Luo Y, Guo L, Zou Y and Xie C: LncRNA ZNF667-AS1 promotes ABLIM1 expression by adsorbing micro RNA-1290 to suppress nasopharyngeal carcinoma cell progression. Onco Targets Ther 13: 4397-4409, 2020.

39. Yao H, Yang L, Tian L, Guo Y and Li Y: LncRNA MSC-AS1 aggravates nasopharyngeal carcinoma progression by targeting miR-524-5p/nuclear receptor subfamily 4 group A member 2 (NR4A2). Cancer Cell Int 20: 138, 2020.

40. Wang W, Dong Y, Li X, Pan Y, Du J and Liu D: MicroRNA-431 serves as a tumor inhibitor in breast cancer through targeting FGF9. Oncol Lett 19: 1001-1007, 2020.

41. Huang W, Zeng C, Hu S, Wang L and Liu J: ATG3, a Target of miR-431-5p, promotes proliferation and invasion of colon cancer via promoting autophagy. Cancer Manag Res 11: 10275-10285, 2019.

42. An G, Ng AY, Meka CS, Luo G, Bright SP, Cazares L, Wright GL Jr and Veltri RW: Cloning and characterization of UROC28, a novel gene overexpressed in prostate, breast, and bladder cancers. Cancer Res 60: 7014-7020, 2000.

43. Pan T, Wu R, Liu B, Wen H, Tu Z, Guo J, Yang J and Shen G: PBOV1 promotes prostate cancer proliferation by promoting G1/S transition. Onco Targets Ther 9: 787-795, 2016.

44. Guo Y, Wu Z, Shen S, Guo R, Wang J, Wang W, Zhao K, Kuang M and Shuai X: Nanomedicines reveal how PBOV1 promotes hepatocellular carcinoma for effective gene therapy. Nat Commun 9: 3430, 2018

45. Carleton NM, Zhu G, Gorbounov M, Miller MC, Pienta KJ, Resar LMS and Veltri RW: PBOV1 as a potential biomarker for more advanced prostate cancer based on protein and digital histomorphometric analysis. Prostate 78: 547-559, 2018.

46. Liu Z, Wu K, Wu J, Tian D, Chen Y, Yang Z and Wu A: NEAT1 is a potential prognostic biomarker for patients with nasopharyngeal carcinoma. J Cell Biochem 120: 9831-9838, 2019.

47. Yao L, Wang T and Wang X: LncRNA FOXP4-AS1 serves as a biomarker for nasopharyngeal carcinoma diagnosis and prognosis. 3 Biotech 11: 25, 2021.

This work is licensed under a Creative Commons

Attribution-NonCommercial-NoDerivatives 4.0 International (CC BY-NC-ND 4.0) License. 